\title{
Caspase-3-resistant uncleavable form of acidic leucine-rich nuclear phosphoprotein 32B potentiates leukemic cell apoptosis
}

\author{
CAI-XIA LI, SHAO-MING SHEN, LI-SHUN WANG and YUN YU
}

\begin{abstract}
Key Laboratory of Cell Differentiation and Apoptosis of Chinese Ministry of Education, Center of Molecular Medicine, Rui-Jin Hospital, Shanghai Jiao-Tong University School of Medicine, Shanghai 200025, P.R. China
\end{abstract}

Received March 6, 2014; Accepted November 14, 2014

DOI: $10.3892 / \mathrm{mmr} .2014 .3035$

\begin{abstract}
One member of the highly conserved acidic leucine-rich nuclear phosphoprotein $32 \mathrm{kDa}$ (ANP32) family of proteins, ANP32B, is critical for normal development, as demonstrated by a study in ANP32B-deficient mice. Another study indicated that ANP32B was a direct substrate of caspase-3, and was primarily cleaved at the sequence Ala-Glu-Val-Asp, following Asp-163. To investigate the significance of ANP32B cleavage in apoptosis, leukemic U937T cell lines were generated with inducible expression of ANP32B(wild type; WT), the uncleavable mutant ANP32B(D163A) and the N-terminal fragment ANP32B(1-163). Notably, overexpression of ANP32B(WT) and ANP32B(D163A) moderately increased and significantly enhanced etoposide-induced apoptosis and caspase-3 activation, whereas expression of ANP32B(1-163) produced no effect. Two hypotheses have been generated, which may explain the distinct roles of the various ANP32B forms: i) ANP32B(WT) and ANP32B(D163A) localize in the nucleus while ANP32B(1-163) mainly resides in the cytosol; or ii) ANP32B(WT) and ANP32B(D163A), butnot ANP32B(1-163), inhibit the expression of the anti-apoptotic protein $\mathrm{Bcl}-2$. Based on these observations, caspase-3-resistant uncleavable ANP32B(D163A) is hypothesized to be pro-apoptotic in leukemic cells.
\end{abstract}

\section{Introduction}

Apoptosis is an indispensable process in embryonic development and tissue homeostasis (1) that is tightly regulated by caspase-mediated signaling cascades. Caspases are part of a family of cysteine proteases that participate in the cleavage of aspartic acid-containing motifs (2). Among the upstream initiator and downstream effector caspases, caspase- 3 is

Correspondence to: Professor Yun Yu, Key Laboratory of Cell Differentiation and Apoptosis of Chinese Ministry of Education, Center of Molecular Medicine, Rui-Jin Hospital, Shanghai Jiao-Tong University School of Medicine, 280 Chong-Qing South Road, Shanghai 200025, P.R. China

E-mail: yy@shsmu.edu.cn

Key words: ANP32B, caspase-3, apoptosis the executioner caspase that triggers the cellular processes resulting in the deconstruction of the cell (3). Various kinases and signaling adaptor proteins have been suggested to be specific substrates of caspase-3, thus it can be inferred that multiple aspects of cell death and survival may be mediated through the cleavage of various signaling molecules $(4,5)$.

The highly conserved acidic nuclear phosphoprotein $32 \mathrm{kDa}$ (ANP32) family of proteins are characterized by an N-terminal leucine-rich repeat (LRR) domain and a C-terminal low-complexity acidic region (LCAR) (6). These proteins have been demonstrated to be involved in a wide array of physiological processes, including cell apoptosis (7-12), differentiation (13-16) and proliferation (17-19). The majority of studies on the ANP32 family have focused on ANP32A, which has been implicated in a variety of cellular functions. For example, it has been demonstrated to enhance caspase- 9 activation via the promotion of apoptosome formation by mediating the oncoprotein prothymosin (9). Additionally, the apoptotic activity of ANP32A has been observed to be crucial in tumor suppression (10), and it contributes to the apoptotic response to chemotherapy in advanced non-small-cell lung cancer (8). ANP32A-deficient mice are, however, viable and fertile with no clear abnormalities, indicating its functional redundancy, which may be due to an overlap of functions with closely related family members (20). Compared with the effects of ANP32A knockout, ANP32B knockout mice display a partially penetrant perinatal lethality, suggesting a hierarchy of importance for the mammalian ANP32 genes, with ANP32B as the most critical for normal development (21).

In a previous study, ANP32B was observed to be a direct substrate of caspase-3 and primarily cleaved at the sequence of Ala-Glu-Val-Asp, following Asp-163. The reduced expression of endogenous ANP32B enhances caspase-3 activation and apoptosis (12). In the current study, in order to determine the functional significance of ANP32B cleavage, leukemic U937T cell lines with inducible expression of ANP32B(wild type; WT), the uncleavable mutant ANP32B(D163A) and the N-terminal fragment ANP32B(1-163) were generated, and the effect of ANP32B cleavage on leukemic cell apoptosis was investigated.

\section{Materials and methods}

Cell lines. The U937T cells provided by Dr Tenen from the Harvard Institute of Medicine (Harvard Medical School, 
Boston, MA, USA) (22) were U937 cells stably transfected with a pUHD-tetracycline-responsive transcription activator (tTA) under the control of a tetracycline-inducible promoter. The U937T cells were cultured in RPMI-1640 medium supplemented with $10 \%$ fetal bovine serum (FBS), $1 \mathrm{mg} / \mathrm{ml}$ tetracycline and $0.5 \mathrm{mg} / \mathrm{ml}$ puromycin (Sigma-Aldrich, St. Louis, MO, USA).

Establishment of U937T stable transformant. Full-length (wild type and D163A mutant) and fragment (1-163) of ANP32B cDNA with flag tag, a polypeptide protein tag with sequence motif DYKXXD, were amplified from the plasmid which was generated as previously described (12), and then cloned and inserted into the pTRE2hyg expression vector (BD Clontech, Palo Alto, CA, USA), a tetracycline-responsive expression vector, in order to form the corresponding plasmids. The sequence of the cDNA insert of the plasmid was confirmed by sequencing (Applied Biosystems 3730/3730xl DNA analyzer, Sangon Biotechnology, Shanghai, China). Following which, the plasmids were transfected into the U937T cells at passage 5 (provided by Dr DG Tenen at Harvard Institutes of Medicine, Harvard Medical School, Boston, MA, USA) containing stably transfected pUHD-tTA (BD Clontech, Palo Alto, CA, USA), whose expression is turned off in the presence of tetracycline (Calbiochem, San Diego, CA). In principle, the expression of tetracycline-responsive transcription activator (tTA) and target proteins should be extremely low in tetracycline-containing medium. In the absence of tetracycline, tTA activates its own promoter to produce more tTA which in turn induces the expression of target proteins (23). To generate the corresponding stable transformants, $1 \times 10^{7}$ U937T cells were washed in RPMI-1640 medium and resuspended in $0.2 \mathrm{ml}$ of Isceve's modified Dulbecco's medium (Sigma-Aldrich) without FBS. A total of $20 \mu \mathrm{g}$ plasmid in $20 \mathrm{ml}$ double-distilled $\mathrm{H}_{2} \mathrm{O}$ was transferred to an electroporation cuvette with a $0.4-\mathrm{cm}$ gap (Bio-Rad Laboratories, Hercules, CA, USA). Electroporation was performed using a Gene Pulser II Electroporation system (Bio-Rad Laboratories) at $170 \mathrm{~V}$ and $960 \mathrm{mF}$. The samples were then transferred to complete RPMI-1640 medium. Subsequent to a $24-\mathrm{h}$ resting period, $1 \mathrm{mg} / \mathrm{ml}$ tetracycline, $0.5 \mathrm{mg} / \mathrm{ml}$ puromycin and $500 \mathrm{mg} / \mathrm{ml}$ hygromycin B (Clontech Laboratories, Inc.) were added and cells were incubated at $37^{\circ} \mathrm{C}$ in $5 \% \mathrm{CO}_{2}$. The positive polyclonal population (pool) was identified based on the induction of the corresponding protein expression following tetracycline withdrawal, and were named U937 $7^{\mathrm{ANP} 32 \mathrm{~B}(\mathrm{WT})}$, U937 ${ }^{\mathrm{ANP32B(D163A)}}$ and U937 ${ }^{\mathrm{ANP} 32 \mathrm{~B}(1-163)}$. An empty plasmid-transfected cell line called U937 $7^{\text {mpty }}$ was used as the control. All cells were maintained in RPMI-1640 medium supplemented with $10 \% \mathrm{FBS}, 1 \mathrm{mg} / \mathrm{ml}$ tetracycline, $0.5 \mathrm{mg} / \mathrm{ml}$ puromycin and $0.5 \mathrm{mg} / \mathrm{ml}$ hygromycin $\mathrm{B}$.

Apoptosis assay. To induce apoptosis, $\sim 3 \times 10^{5}$ cells $/ \mathrm{ml}$ were initially seeded and cultured in tetracycline-free Isceve's modified Dulbecco's medium for 10 days to induce the expression of the corresponding proteins. The cells were then incubated with $1 \mu \mathrm{M}$ etoposide (Enzo Life Sciences, Inc., Farmingdale, NY, USA) for an additional $12 \mathrm{~h}$. The annexin-V assay was performed using a flow cytometer (BD FACSCanto II; BD Biosciences, San Jose, CA, USA) according to the manufacturer's instructions for the ApoAlert Annexin-V Apoptosis kit (Clontech Laboratories, Inc.), in order to count the number of annexin-V-positive apop- totic cells. Caspase-3 activity was determined by the Caspase 3 Assay kit, Colorimetric (Sigma-Aldrich).

Western blots. The protein lysates were equally loaded onto a 10-12\% SDS-PAGE gel using a mini gel apparatus (Bio-Rad Laboratories, Inc.) and subsequently transferred to a nitrocellulose membrane (Bio-Rad Laboratories). The membranes were blocked with 5\% nonfat dry milk solution in tris-buffered saline (Beyotime) for $1 \mathrm{~h}$ at room temperature and then incubated in primary antibody dissolved in the $5 \%$ nonfat dry milk solution at $4^{\circ} \mathrm{C}$ overnight. The following antibodies were used:

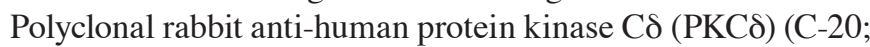
SC-937; Santa Cruz Biotechnology, Inc., Santa Cruz, CA, USA); polyclonal rabbit anti-human cleaved caspase-3 (9661; Cell Signaling Technology, Danvers, MA, USA); monoclonal mouse anti-human poly[ADP (adenosine diphosphate)-ribose] polymerase (PARP; F-2; SC-8007; Santa Cruz Biotechnology, Inc.); polyclonal rabbit anti-human Bcl-2 (sc-492; Santa Cruz Biotechnology, Inc.) and polyclonal rabbit anti-human ANP32B (10843-1-AP; ProteinTech Group, Inc., Chicago, IL, USA), with monoclonal mouse anti- $\beta$-actin mAb (EMD Millipore, Billerica, MA, USA) to confirm equal loading. Following washing with PBS, the blots were incubated with horseradish peroxidase-conjugated secondary antibody (Dako, Glostrup, Denmark) corresponding to the primary antibody in $5 \%$ nonfat dry milk solution for $1 \mathrm{~h}$ at room temperature. Proteins were then detected using a luminol detection reagent (Santa Cruz Biotechnology, Inc.), and developed onto X-ray films (Kodak, Rochester, NY, USA).

Immunofluorescent staining of cells. Cells were collected onto slides using a Shandon Cytospin 4 Cytocentrifuge (Thermo Fisher Scientific, Waltham, MA, USA) at $800 \mathrm{x} g$ for $5 \mathrm{~min}$ and fixed with $4 \%$ formaldehyde (Beyotime) for $10 \mathrm{~min}$. Subsequent to permeabilization with $0.3 \%$ Triton $\mathrm{X}-100$ (Beyotime) in phosphate-buffered saline (PBS; Beyotime) for $10 \mathrm{~min}$, cells were incubated with $1 \%$ bovine serum albumin (Beyotime) in PBS for $2 \mathrm{~h}$ at room temperature, followed by overnight incubation with the ANP32B antibody. Cells were stained with fluorescein isothiocyanate-labeled anti-rabbit IgG (Dako) for $1 \mathrm{~h}$. Cell nuclei were stained with 4',6-diamidino-2-phenylindole (Molecular Probes Life Technologies, Eugene, OR, USA). Fluorescent signals were measured using an MRC-1024 laser scanning confocal microscope equipped with a Zeiss x60 objective (Bio-Rad Laboratories) or an Olympus BX51 fluorescence microscope (100x/1.30 oil objective lens; Olympus Corporation, Tokyo, Japan).

Statistical analysis. The differences between the two groups were analyzed for statistical significance using the nonparametric Wilcoxon rank sum test. $\mathrm{P}<0.05$ was considered to indicate a statistically significant difference.

\section{Results}

Establishment of cell lines with inducible expression of ANP32B and its mutants. In previous studies, ANP32B has been demonstrated to be a caspase-3 substrate during apoptotic induction, and is primarily cleaved after Asp163 (12). To determine the functional significances of ANP32B cleavage during apoptosis, 
A

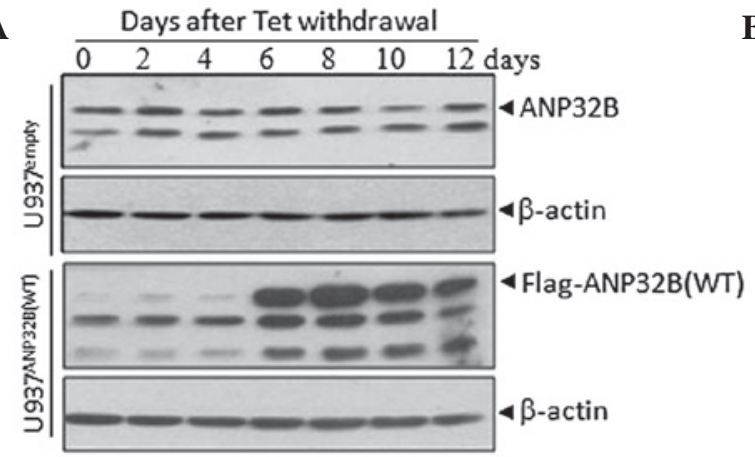

B

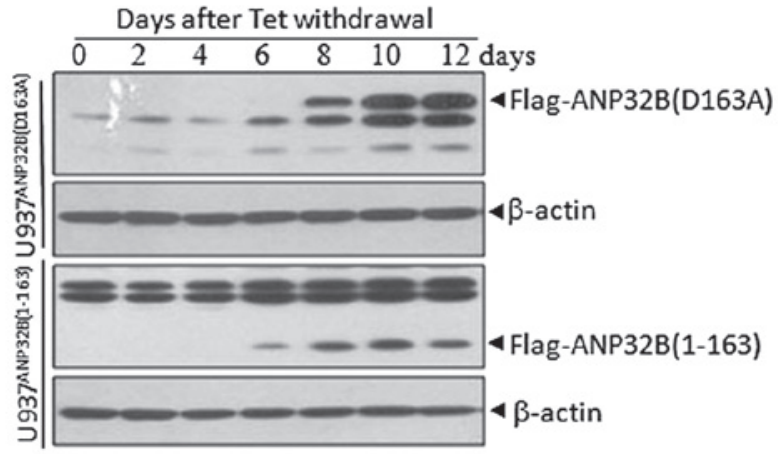

Figure 1. Inducible forms of ANP32B expression in the corresponding transformant cells following Tet withdrawal. The (A) U937T ${ }^{\mathrm{mpty}}$, U937T $\mathrm{T}^{\mathrm{ANP} 32 \mathrm{~B}(\mathrm{WT})}$ and

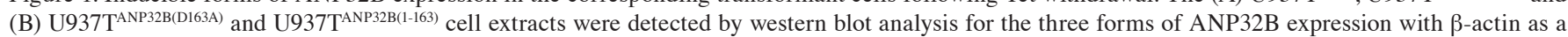
loading control. ANP32B, acidic leucine-rich nuclear phosphoprotein 32B; Tet, tetracycline, WT, wild type.
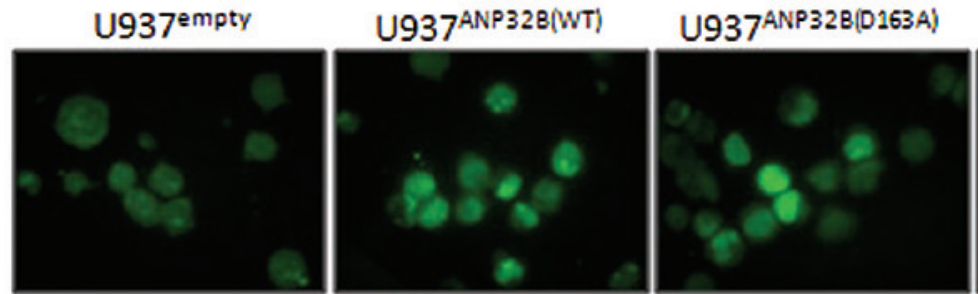

U937 ANP32B(1-163)
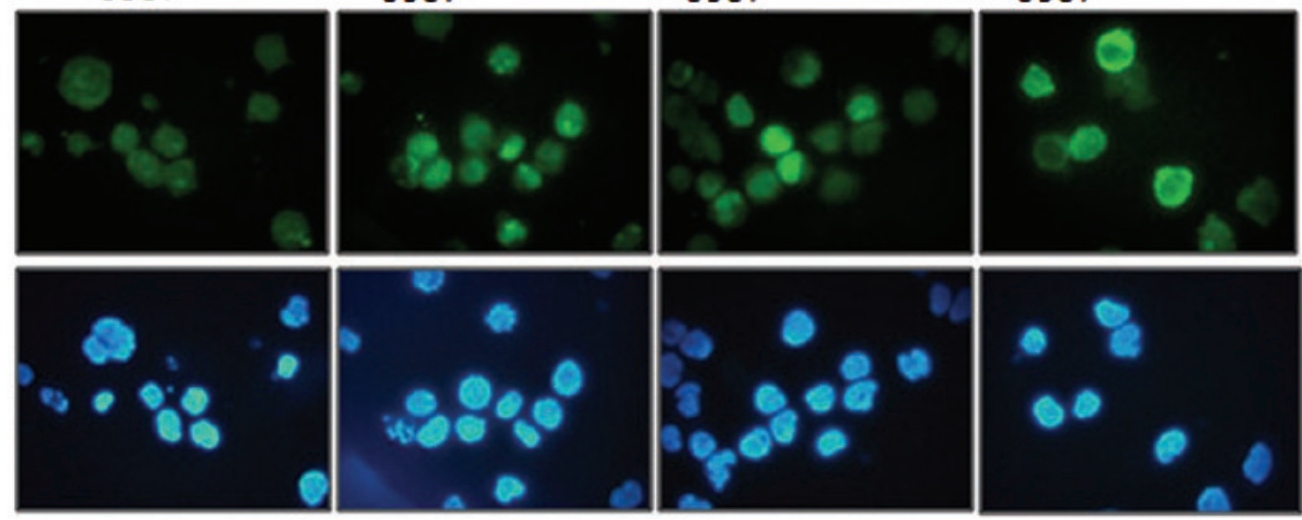

anti-ANP32B

Figure 2. The localization of the different ANP32B forms in the transformant cells following tetracycline withdrawal immunostained with ANP32B antibody in addition to duplicate staining of 4',6-diamidino-2-phenylindole. Images are representative of $>20$ fields examined. ANP32B, acidic leucine-rich nuclear phosphoprotein 32B; WT, wild type.

A

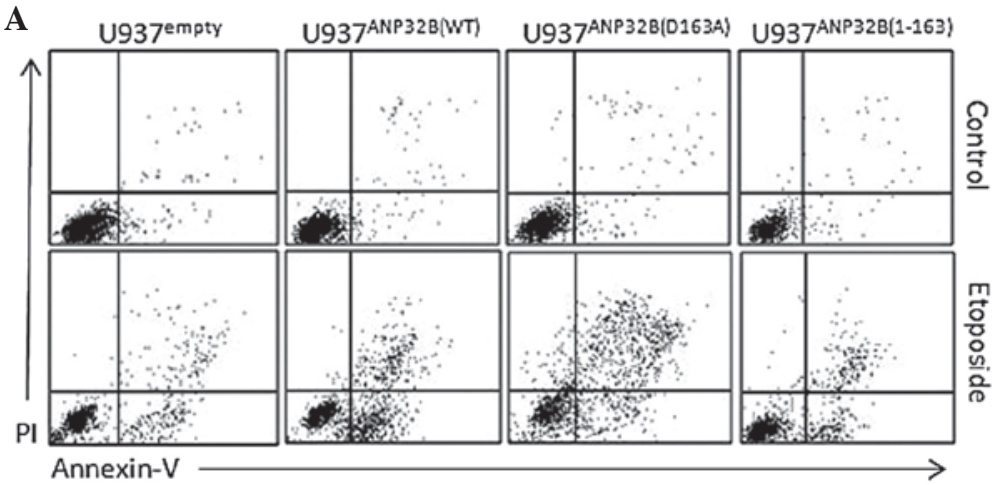

B

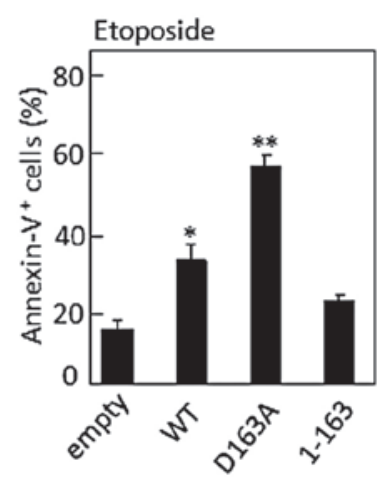

Figure 3. Uncleavable ANP32B(D163A) protein enhances etoposide-induced apoptosis. (A) Percentage of annexin- $\mathrm{V}^{+}$cells was determined using flow cytometry, and (B) displayed in a histogram. All values are presented as the mean \pm standard deviation; experiments were performed in triplicate. ${ }^{*}<0.05$ and ${ }^{* *} \mathrm{P}<0.01$ vs. U937T ${ }^{\text {empty }}$ cells. ANP32B, acidic leucine-rich nuclear phosphoprotein 32B; WT, wild type; PI. propidium iodide.

leukemic U937T cell lines with inducible expression of WT, D163A mutant and N-terminal fragment (1-163) ANP32B, were generated by a Tet-Off gene expression system. In the Tet-off system, gene expression is turned on when tetracycline is removed from the culture medium. U937T ${ }^{\text {empty }}$, U937T ${ }^{\mathrm{ANP} 32 \mathrm{~B}(\mathrm{WT})}$, $\mathrm{U} 37 \mathrm{~T}^{\mathrm{ANP} 32 \mathrm{~B}(\mathrm{D} 163 \mathrm{~A})}$ and U937T $\mathrm{T}^{\mathrm{ANP32B(1-163)}}$ cells were all induced to express flag-tagged ANP32B or its mutants, by tetracycline withdrawal for 6-12 days (Fig. 1). Immunofluorescent analysis with the antibody against ANP32B indicated that ANP32B(WT) and $\mathrm{ANP} 32 \mathrm{~B}(\mathrm{D} 163 \mathrm{~A})$ proteins were localized in the nuclei, while ANP32B(1-163) was in the cytosol (Fig. 2). This was due to the absence of the nuclear localization signal (NLS), which was mapped to amino acid residues KRKR at position 239-242 in the C-terminal of this protein. 


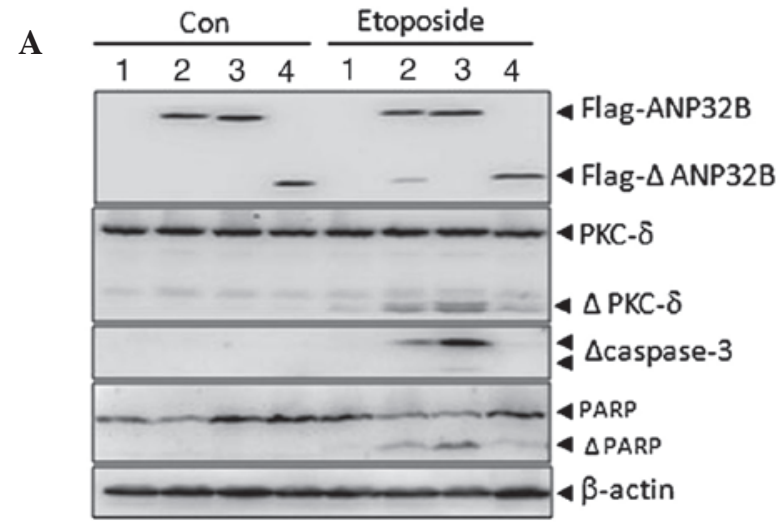

$\mathbf{B}$

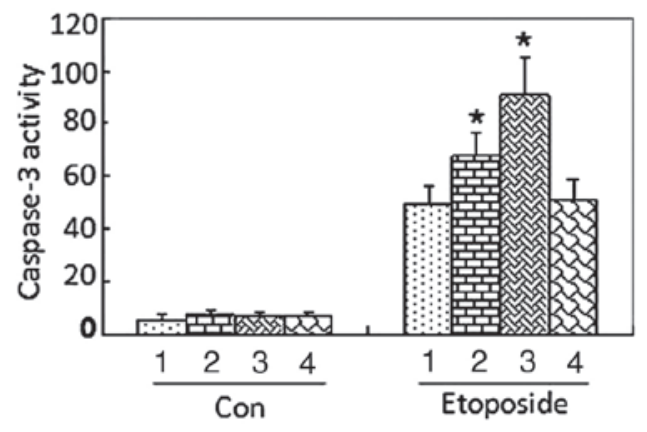

Figure 4. Effect of uncleavable ANP32B(D163A) protein on etoposide-induced proteolytic cleavage of PKCd and caspase-3 activation. (A) Western blot analysis with $\beta$-actin as a loading control. (B) Analysis of caspase-3 activity. ${ }^{*} \mathrm{P}<0.05$ vs. U937T ${ }^{\text {empty }}$ cells with the corresponding treatment in an independent experiment with triplicates. ANP32B, acidic leucine-rich nuclear phosphoprotein 32B; PKC- $\delta$, protein kinase C $\delta$; WT, wild type; $1, \mathrm{U}^{2} 37 \mathrm{~T}^{\mathrm{empty}} ; 2, \mathrm{U} 937 \mathrm{~T}^{\mathrm{ANP} 32 \mathrm{~B}(\mathrm{WT})} ; 3$, $\mathrm{U} 37 \mathrm{~T}^{\mathrm{ANP} 32 \mathrm{~B}(\mathrm{D} 163 \mathrm{~A})} ; 4$, U937T ${ }^{\mathrm{ANP} 32 \mathrm{~B}(1-163)}$; PARP, poly[ADP (adenosine diphosphate)-ribose] polymerase; Con, control; $\Delta$, fragments of the corresponding protein,
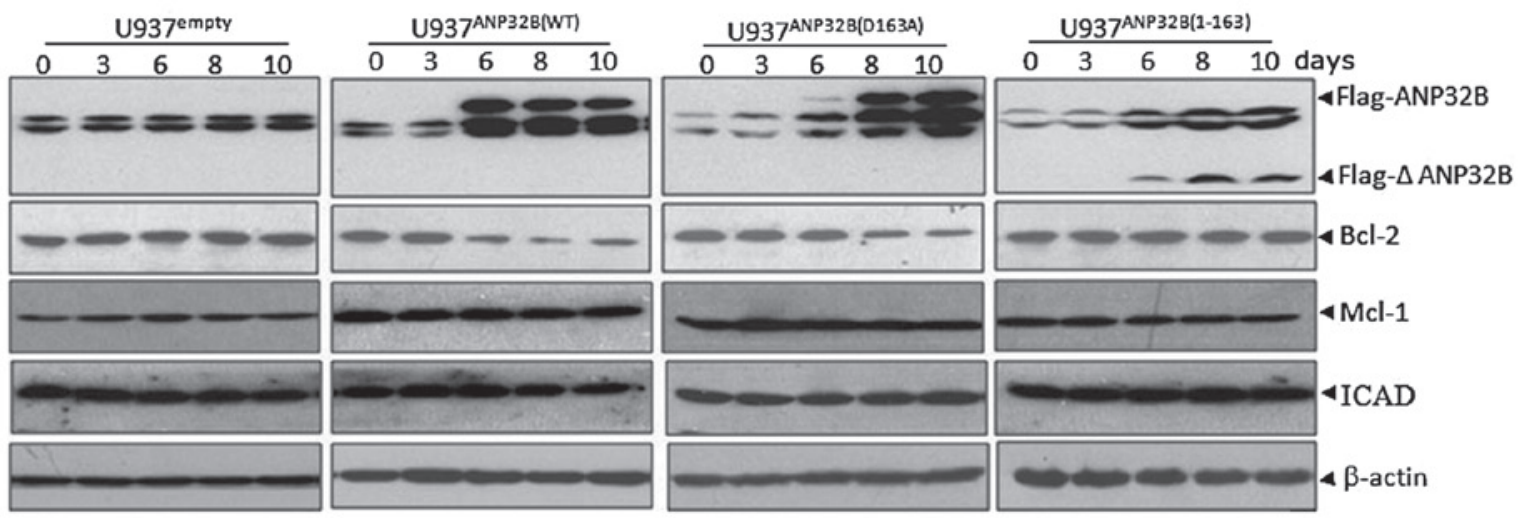

Figure 5. Uncleavable ANP32B(D163A) induction reduces Bcl-2 protein expression levels. U937T ${ }^{\text {empty }}, \mathrm{U} 937 \mathrm{~T}^{\mathrm{ANP} 32 \mathrm{~B}(\mathrm{WT})}$, U937T ${ }^{\mathrm{ANP} 32 \mathrm{~B}(\mathrm{D} 163 \mathrm{~A})}$ and U937T ${ }^{\mathrm{ANP} 32 \mathrm{~B}(1-163)}$ cells were cultured in the absence of tetracycline for different time periods as indicated. Bcl-2, Mcl-1 and ICAD proteins were probed by western blot analysis and $\beta$-actin was used as a loading control. ANP32B, acidic leucine-rich nuclear phosphoprotein 32B; ICAD, inhibitor of caspase-activated DNase; WT, wild type.

Uncleavable ANP32B(D163A) protein amplifies apoptosis induction via caspase-3. The contribution of caspase-3-mediated cleavage of ANP32B to apoptosis was investigated by inducing the above cell lines to undergo apoptosis and measuring the number of annexin-V-positive cells using flow cytometry (Fig. 3A). The results demonstrated that compared with U937T $T^{\text {empty }}$ cells $(18.3 \pm 1.9 \%)$, apoptosis was significantly increased in $\mathrm{U} 37 \mathrm{~T}^{\mathrm{ANP} 32 \mathrm{~B}(\mathrm{WT})}$ cells $(37.2 \pm 2.7 \% ; \mathrm{P}<0.05)$ as well as highly significantly enhanced in U937 $\mathrm{T}^{\mathrm{ANP} 32 \mathrm{~B}(\mathrm{D} 163 \mathrm{~A})}$ cells $(58.5 \pm 2.1 \% ; \mathrm{P}<0.01)$, but was not markedly altered in U937T ${ }^{\mathrm{ANP} 32 \mathrm{~B}(1-163)}$ cells $(21.8 \pm 0.8 \%)$ (Fig. 3B). This suggested that the uncleavable ANP32B(D163A) possesses pro-apoptotic activity.

Uncleavable ANP32B(D163A) protein enhances PKC 8 and caspase-3 activity. The catalytic activity of PKC $\delta$ is established to be important at the early stage of etoposide-induced apoptosis (prior to caspase-3 activation) $(24,25)$, while activated caspase- 3 produces an amplifying effect on PKC $\delta$ activation (26). To further confirm the roles of different ANP32B forms in apoptosis, the proteolytic activation of PKC $\delta$ and caspase- 3 was investigated, in addition to the cleavage of PARP, a common substrate of activated caspase-3 during apoptosis. As depicted in Fig. 4A, treatment with $1 \mu \mathrm{M}$ etoposide induced slight fragmentation of $\mathrm{PKC} \delta$, caspase-3 and PARP in U937 $T^{\text {Tmpty }}$ cells, while these fragmentations were moderately and significantly enhanced in $\mathrm{U} 337 \mathrm{~T}^{\mathrm{ANP} 32 \mathrm{~B}(\mathrm{WT})}$ and $\mathrm{U} 3 \mathrm{~T}^{\mathrm{ANP}}{ }^{\mathrm{N} 2 \mathrm{~B}(\mathrm{D} 163 \mathrm{~A})}$ cells, respectively, but remained unchanged in U937T ${ }^{\mathrm{ANP} 2 \mathrm{~B}(1-163)}$ cells. Caspase-3 activation was observed to be regulated similarly (Fig. 4B).

Inducible expression of uncleavable ANP32B(D163A) protein reduces Bcl-2 protein levels. ANP32B has been reported to regulate gene transcription as a histone chaperone in the nucleus (27), thus, the mechanisms underlying the pro-apoptotic role of full-length ANP32B were investigated, via the detection of several key apoptosis-associated genes, including Bcl-2, Mcl-1 and inhibitor of caspase-activated DNase (ICAD) in the inducible cell lines. The data indicated that the expression levels of anti-apoptotic Bcl-2 protein were progressively reduced subsequent to tetracycline removal, in parallel with the induction of full-length ANP32B protein in $\mathrm{U}^{2} 37 \mathrm{~T}^{\mathrm{ANP} 32 \mathrm{~B}(\mathrm{WT})}$ and U937 $\mathrm{T}^{\mathrm{ANP} 32 \mathrm{~B}(\mathrm{D} 163 \mathrm{~A})}$ cells, while Mcl-1 and ICAD expression remained unchanged (Fig. 5). In addition, the expression of $\mathrm{Bcl}-2$ 
was not affected by ANP32B(1-163), which was localized in the cytosol and had no effect on apoptosis induction. The reduced expression of $\mathrm{Bcl}-2$ protein is hypothesized to contribute to the apoptosis-enhancing ability of full-length ANP32B.

\section{Discussion}

Based on previous subcellular proteomic analysis (28), ANP32B was identified as a novel caspase-3 substrate primarily cleaved at the sequence of Ala-Glu-Val-Asp, following Asp-163 (12). In order to ascertain whether the cleavage of ANP32B contributes to apoptosis induction in leukemic cells, leukemic U937T cell lines with inducible expression of ANP32B(WT) along with two mutants, the D163A mutant and its N-terminal fragment (1-163) were generated, which were treated with etoposide following tetracycline withdrawal. Notably, overexpression of either ANP32B(WT) or its uncleavable mutant [ANP32B(D163A)] was observed to accelerate etoposide-induced apoptosis and caspase-3 activation, whereas enforced expression of the N-terminal fragment [ANP32B(1-163)] had no effect, suggesting the LCAR of ANP32B may be critical for the apoptosis-inducing activity of this protein. A previous study that supports this theory demonstrated that ANP32A LCAR is able to directly promote caspase-3 activation (29). In addition, the cellular localization of ANP32B(1-163) lacking LCAR was different from ANP32B(WT) and ANP32B(D163A). Consistent with the study by Mutai et al (30), the current study demonstrated that full length ANP32B protein (WT and D163A mutant) accumulated in the nucleus, whereas the N-terminal fragment of ANP32B was localized in the cytoplasm, due to the absence of the NLS. This may be the primary cause of the varied apoptosis-inducing abilities of the three transformants.

An anti-apoptotic function of ANP32B has been previously reported, indicating that siRNA-mediated depletion of ANP32B enhanced NSC606985- and etoposide-induced apoptosis (12). However, the current study observed that overexpression of ANP32B(WT) also enhanced etoposide-induced apoptosis and the uncleavable ANP32B(D163A) mutant significantly accelerated it, suggesting a pro-apoptotic function of ANP32B. However, the explanation of this inconsistency remains unclear. It is hypothesized that ANP32B may have bidirectional roles in apoptotic induction. In support of this theory, nuclear factor- $\kappa \mathrm{B}$ has been reported to exhibit anti-apoptotic and pro-apoptotic activity, via the induction of different anti-apoptotic and pro-apoptotic genes (31). From the observation that ANP32B was reported to regulate transcription factor activity as a histone chaperone, it may be hypothesized that the bidirectional roles of ANP32B potentially result from its regulation of certain transcription factors with bidirectional roles in apoptosis induction.

To further investigate the mechanism of the pro-apoptotic activity of full-length ANP32B, the expression of several apoptosis-associated genes were analyzed in these inducible cell lines. As demonstrated in Fig. 5, the expression of ANP32B(WT) and its uncleavable mutant [ANP32B(D163A)] resulted in progressively reduced expression of the anti-apoptotic Bcl-2 protein, suggesting that ANP32B may exert its pro-apoptotic role via the inhibition of Bcl-2 expres- sion. However, the mechanism of regulation of the $\mathrm{Bcl}-2$ protein remains unclear and requires further investigation.

\section{Acknowledgements}

The current study was supported by the Ministry of Science and Technology (grant no. 2013CB910903), the Shanghai Science and Technology Committee (grant no. 11ZR1421500) and the National Natural Science Foundation of China (grant nos. 30971276, 31170783 and 31300679).

\section{References}

1. Fuchs Y and Steller H: Programmed cell death in animal development and disease. Cell 147: 742-758, 2011.

2. Thornberry NA and Lazebnik Y: Caspases: enemies within. Science 281: 1312-1316, 1998.

3. Utz PJ and Anderson P: Life and death decisions: regulation of apoptosis by proteolysis of signaling molecules. Cell Death Differ 7: 589-602, 2000.

4. Johnson CE and Kornbluth S: Caspase cleavage is not for everyone. Cell 134: 720-721, 2008.

5. Timmer JC and Salvesen GS: Caspase substrates. Cell Death Differ 14: 66-72, 2007.

6. Matilla A and Radrizzani M: The Anp32 family of proteins containing leucine-rich repeats. Cerebellum 4: 7-18, 2005.

7. Adegbola $\mathrm{O}$ and Pasternack GR: Phosphorylated retinoblastoma protein complexes with pp32 and inhibits pp32-mediated apoptosis. J Biol Chem 280: 15497-15502, 2005.

8. Hoffarth S, Zitzer A, Wiewrodt R, et al: pp32/PHAPI determines the apoptosis response of non-small-cell lung cancer. Cell Death Differ 15: 161-170, 2008.

9. Jiang X, Kim HE, Shu H, et al: Distinctive roles of PHAP proteins and prothymosin-alpha in a death regulatory pathway. Science 299: 223-226, 2003.

10. Pan W, da Graca LS, Shao Y, et al: PHAPI/pp32 suppresses tumorigenesis by stimulating apoptosis. J Biol Chem 284: 6946-6954, 2009.

11. Schafer ZT, Parrish AB, Wright KM, et al: Enhanced sensitivity to cytochrome c-induced apoptosis mediated by PHAPI in breast cancer cells. Cancer Res 66: 2210-2218, 2006.

12. Shen SM, Yu Y, Wu YL, et al: Downregulation of ANP32B, a novel substrate of caspase-3, enhances caspase-3 activation and apoptosis induction in myeloid leukemic cells. Carcinogenesis 31: 419-426, 2010.

13. Brody JR, Kadkol SS, Hauer MC, et al: pp32 reduction induces differentiation of TSU-Prl cells. Am J Pathol 164: 273-283, 2004.

14. Kular RK, Cvetanovic M, Silferd S et al: Neuronal differentiation is regulated by leucine-rich acidic nuclear protein (LANP), a member of the inhibitor of histone acetyltransferase complex. J Biol Chem 284: 7783-7792, 2009.

15. Puente LG, Carrière JF, Kelly JF and Megeney LA: Comparative analysis of phosphoprotein-enriched myocyte proteomes reveals widespread alterations during differentiation. FEBS Lett 574: 138-144, 2004.

16. Yu Y, Shen SM, Zhang FF, et al: Acidic leucine-rich nuclear phosphoprotein 32 family member B (ANP32B) contributes to retinoic acid-induced differentiation of leukemic cells. Biochem Biophys Res Commun 423: 721-725, 2012.

17. Amasaki H, Ogawa M, Nagasao J, et al: Distributional changes of BrdU, PCNA, E2F1 and PAL31 molecules in developing murine palatal rugae. Ann Anat 185: 517-523, 2003.

18. Sun W, Hattori N, Mutai H, et al: PAL31, a nuclear protein required for progression to the $\mathrm{S}$ phase. Biochem Biophys Res Commun 280: 1048-1054, 2001.

19. Fukukawa C, Tanuma N, Okada T, et al: pp32/ I-1(PP2A) negatively regulates the Raf-1/MEK/ERK pathway. Cancer Lett 226: $155-160,2005$.

20. Opal P, Garcia JJ, McCall AE, et al: Generation and characterization of LANP/pp32 null mice. Mol Cell Biol 24: 3140-3149, 2004.

21. Reilly PT, Afzal S, Gorrini C, et al: Acidic nuclear phosphoprotein $32 \mathrm{kDa}$ (ANP32)B-deficient mouse reveals a hierarchy of ANP32 importance in mammalian development. Proc Natl Acad Sci USA 108: 10243-10248, 2011. 
22. Boer J, Bonten-Surtel J and Grosveld G: Overexpression of the nucleoporin CAN/NUP214 induces growth arrest, nucleocytoplasmic transport defects, and apoptosis. Mol Cell Biol 18: $1236-1247,1998$

23. Boer J, Bonten-Surtel J and Grosveld G: Overexpression of the nucleoporin CAN/NUP214 induces growth arrest, nucleocytoplasmic transport defects, and apoptosis. Mol Cell Biol 18: 1236-1247, 1998

24. Matassa AA, Carpenter L, Biden TJ, et al: PKCdelta is required for mitochondrial-dependent apoptosis in salivary epithelial cells. J Biol Chem 276: 29719-29728, 2001.

25. Qi X and Mochly-Rosen D: The PKCdelta -Abl complex communicates ER stress to the mitochondria - an essential step in subsequent apoptosis. J Cell Sci 121: 804-813, 2008.

26. Song MG, Gao SM, Du KM, et al: Nanomolar concentration of NSC606985, a camptothecin analog, induces leukemic-cell apoptosis through protein kinase Cdelta-dependent mechanisms. Blood 105: 3714-3721, 2005.
27. Munemasa Y,Suzuki T, Aizawa K, et al: Promoter region-specific histone incorporation by the novel histone chaperone ANP32B and DNA-binding factor KLF5. Mol Cell Biol 28: 1171-1181, 2008.

28. Yu Y, Wang LS, Shen SM, et al: Subcellular proteome analysis of camptothecin analogue NSC606985-treated acute myeloid leukemic cells. J Proteome Res 6: 3808-3818, 2007.

29. Hill MM, Adrain C, Duriez PJ, et al: Analysis of the composition, assembly kinetics and activity of native Apaf-1 apoptosomes. EMBO J 23: 2134-2145, 2004.

30. Mutai H, Toyoshima Y, Sun W, et al: PAL31, a novel nuclear protein, expressed in the developing brain. Biochem Biophys Res Commun 274: 427-433, 2000.

31. Karin M and Lin A: NF-kappaB at the crossroads of life and death. Nat Immunol 3: 221-227, 2002. 